\title{
Speckle Reduction and Contrast Enhancement of Echocardiograms via Multiscale Nonlinear Processing
}

\author{
Xuli Zong,* Andrew F. Laine, and Edward A. Geiser
}

\begin{abstract}
This paper presents an algorithm for speckle reduction and contrast enhancement of echocardiographic images. Within a framework of multiscale wavelet analysis, we apply wavelet shrinkage techniques to eliminate noise while preserving the sharpness of salient features. In addition, nonlinear processing of feature energy is carried out to enhance contrast within local structures and along object boundaries. We show that the algorithm is capable of not only reducing speckle, but also enhancing features of diagnostic importance, such as myocardial walls in two-dimensional echocardiograms obtained from the parasternal short-axis view.

Shrinkage of wavelet coefficients via soft thresholding within finer levels of scale is carried out on coefficients of logarithmically transformed echocardiograms. Enhancement of echocardiographic features is accomplished via nonlinear stretching followed by hard thresholding of wavelet coefficients within selected (midrange) spatial-frequency levels of analysis.

We formulate the denoising and enhancement problem, introduce a class of dyadic wavelets, and describe our implementation of a dyadic wavelet transform. Our approach for speckle reduction and contrast enhancement was shown to be less affected by pseudo-Gibbs phenomena. We show experimentally that this technique produced superior results both qualitatively and quantitatively when compared to results obtained from existing denoising methods alone. A study using a database of clinical echocardiographic images suggests that such denoising and enhancement may improve the overall consistency of expert observers to manually defined borders.
\end{abstract}

Index Terms - Contrast enhancement, denoising, echocardiograms, multiscale representations, nonlinear processing, speckle reduction, ultrasound images, wavelet shrinkage.

\section{INTRODUCTION}

$\mathbf{N}$ OISE and artifacts can cause signal and image degradations for many medical imaging modalities. Different image modalities exhibit distinct types of degradation. Radiographs often exhibit low contrast while images formed with coherent energy, such as ultrasound, suffer from speckle noise. Image degradation can have a significant impact on image quality and thus affect human interpretation and the

Manuscript received March 31, 1997; revised July 22, 1998. The Associate Editor responsible for coordinating the review of this paper and recommending its publication was C. Meyer. Asterisk indicates corresponding author.

*X. Zong was with the Department of Computer and Information Science and Engineering, University of Florida, Gainesville, FL 32611 USA. He is now with GE Corporate Research and Development, P.O. Box 8, Building K-1, Room NMR-136, Schenectady, NY 12301 USA (e-mail: zong@crd.ge.com).

A. F. Laine is with the Center for Biomedical Engineering, Columbia University, New York, NY 10027 USA.

E. A. Geiser is with Echocardiography Research Laboratory, Department of Medicine, University of Florida, Gainesville, FL 32610 USA.

Publisher Item Identifier S 0278-0062(98)08049-5. accuracy of computer-assisted methods. Poor image quality often makes feature extraction, analysis, recognition, and quantitative measurements problematic and unreliable. The promise of image restoration and contrast enhancement has motivated a considerable amount of research in imaging science and medical imaging [1]-[11]. The denoising and feature enhancement techniques developed in this study may help to improve the accuracy and reliability of image processing algorithms targeting both qualitative and quantitative problems.

Image formation under coherent waves results in a granular pattern known as speckle. The granular pattern is correlated with the surface roughness of an object being imaged. In [12], Goodman presented an analysis of speckle properties under coherent irradiance. The primary differences between laser and ultrasound speckle were pointed out by Abbott and Thurstone [13] in terms of coherent interference and speckle production. For speckle reduction, earlier techniques include temporal averaging [12], [13], median filtering, and homomorphic Wiener filtering [1]. Similar to temporal averaging, one speckle reduction technique [14] used frequency and/or angle diversity to generate multiple uncorrelated syntheticaperture radar (SAR) images which were summed incoherently to reduce speckle. Homomorphic Wiener filtering is a method which converts multiplicative noise into additive noise and applies Wiener low-pass filtering to reduce noise. In [15], a coherent image was decomposed into three components, one of which, called subresolvable quasi-periodic scatter, causes speckle noise. The component was eliminated by a harmonic analysis algorithm. An algorithm based on the maximumlikelihood principle and a wavelet regularization procedure for the logarithm of a radar image was also developed to reduce speckle in [16]. A wavelet-based method for speckle reduction was first reported by Guo et al. [5]. In the method of Guo et al., wavelet shrinkage of the logarithmically transformed image is applied to speckle reduction of SAR images. They also proposed several approaches to combine the data from polarization to achieve better performance. During the last two decades, modality specific image enhancement schemes have been developed and studied in the literature [1], [6], [7], [17]. Specifically, various spatial and frequency-based techniques [1], [2], [18], [19] have been developed for ultrasound image enhancement. A method called statistical enhancement [20] used the local standard deviation of a surrounding region centered around each pixel to replace its value to enhance edges in ultrasound images. 
At first glance, denoising and feature enhancement appear to be two conflicting objectives. However, they are simply two sides of the same coin. The purpose of denoising is to eliminate noise, especially those that exist primarily in high-frequency bands, while methods of feature enhancement attempt to enhance specific signal details. The difference is that features often occupy a wider frequency band than noise. It is even more difficult to achieve both objectives when signal details are corrupted by noise. Traditional spatial and filtering-based methods for denoising often reduce noise at the price of blurred features while single-scale conventional methods for contrast enhancement may amplify noise. Single-scale representation of a signal in time (or pure frequency) are problematic when attempting to discriminate signal from noise. In our approach, we achieve denoising and feature enhancement under a framework of multiscale wavelet analysis. We seek to eliminate noise while restoring or enhancing salient features. Through multiscale representation by a discrete dyadic wavelet transform (DWT) with a first-order derivative of a smoothing function as its basis wavelet we can distinguish feature energy from noise reasonably well. The objectives of denoising and feature enhancement are achieved by simultaneously lowering noise energy and raising feature energy through judicious nonlinear processing of wavelet coefficients in the transform domain. Through parameterized processing, we are able to achieve a flexible control and the potential to reduce speckle and restore (or even enhance) contrast along features, such as object boundaries. As in our earlier work [21], this approach for speckle reduction and contrast enhancement is less affected by pseudo-Gibbs phenomena [8]. Our approach is similar to the method reported by Guo et al. [5]. The differences are that 1) different wavelets and multiscale overcomplete representations are used in our approach, and 2) an enhancement mechanism is incorporated into our denoising process.

This paper is organized as follows. Section II presents a finite-level discrete DWT which is formulated in two dimensions. Our method for speckle reduction and contrast enhancement under multiscale wavelet analysis is described in Section III. This includes speckle noise modeling, wavelet shrinkage for noise reduction, nonlinear stretching (feature energy gain) for contrast restoration and enhancement, and the complete algorithm in overview. Section IV presents experimental results, analysis, and discussions. In Section V, we describe a study using clinical images to test the performance of denoising/enhancement on the consistency and reliability to manually defined borders by expert observers. We show via quantitative measurements that borders defined by experts on denoised and feature-enhanced echocardiograms were more consistent than those detected on the corresponding (unprocessed) speckled echocardiograms. Finally, Section VI concludes the paper.

\section{Discrete Dyadic WaVelet TRANSFORM}

The multiscale DWT developed by Mallat and Zhong [22] has been previously applied in areas including edge detection, texture analysis, noise reduction, and image enhancement. A finite-level discrete DWT of a 2-D discrete function $f(m, n) \in$

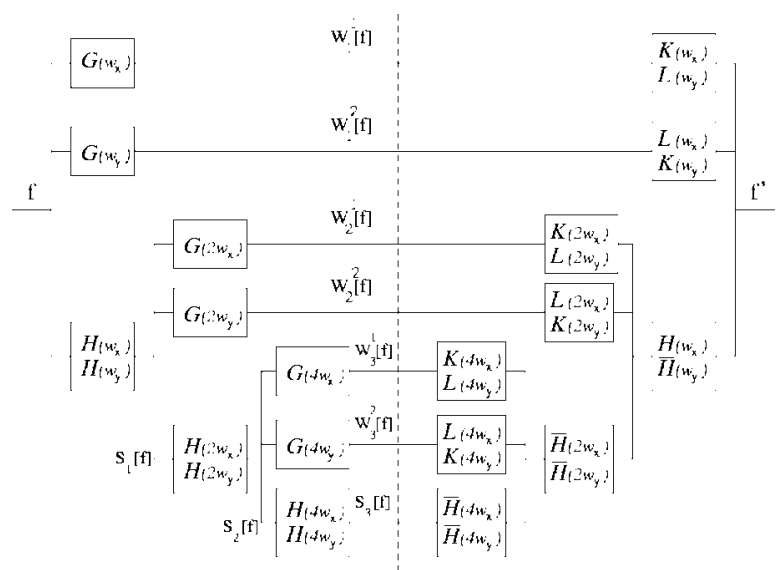

Fig. 1. A 3-level DWT decomposition and reconstruction of a 2-D function.

$\boldsymbol{l}^{2}\left(\boldsymbol{Z}^{2}\right)$ can be represented as

$$
\mathcal{W}[f(m, n)]=\left\{\left(W_{j}^{d}[f(m, n)]\right)_{d=1,2,1 \leq j \leq J}, S_{J}[f(m, n)]\right\}
$$

where $W_{j}^{d}[f(m, n)]$ is a wavelet coefficient at scale $2^{j}$ (or level $j$ ), position $(m, n)$, and spatial orientation $d$ (one for horizontal and two for vertical), $S_{J}[f(m, n)]$ is a coarse scale approximation at the final level $J$ and position $(m, n)$. For details about DWT's, we refer the reader to [22].

The finite-level dyadic wavelet decomposition in (1) forms a complete representation for a J-level DWT. For a particular class of 2-D dyadic wavelets, such as the first-order derivatives of spline smoothing functions, Mallat and Zhong [22] showed that the finite-level direct and inverse discrete DWT of a 2-D discrete function can be implemented in terms of four filters, $H, G, K$, and $L$. The four filters should satisfy the following perfect decomposition and reconstruction conditions:

$$
\begin{aligned}
|H(\omega)|^{2}+G(\omega) K(\omega) & =1 \\
L(\omega) & =\frac{1+|H(\omega)|^{2}}{2} .
\end{aligned}
$$

The dyadic wavelet decomposition in (1) can be formulated in terms of the following recursive relations between the two levels $j$ and $j+1$ in the Fourier domain as

$$
\begin{aligned}
\hat{W}_{j+1}^{1}\left[f\left(\omega_{x}, \omega_{y}\right)\right] & =G\left(2^{j} \omega_{x}\right) \hat{S}_{j}\left[f\left(\omega_{x}, \omega_{y}\right)\right] \\
\hat{W}_{j+1}^{2}\left[f\left(\omega_{x}, \omega_{y}\right)\right] & =G\left(2^{j} \omega_{y}\right) \hat{S}_{j}\left[f\left(\omega_{x}, \omega_{y}\right)\right] \\
\hat{S}_{j+1}\left[f\left(\omega_{x}, \omega_{y}\right)\right] & =H\left(2^{j} \omega_{x}\right) H\left(2^{j} \omega_{y}\right) \hat{S}_{j}\left[f\left(\omega_{x}, \omega_{y}\right)\right]
\end{aligned}
$$

where $j \geq 0$, and $\hat{S}_{0}\left[f\left(\omega_{x}, \omega_{y}\right)\right]:=\hat{f}\left(\omega_{x}, \omega_{y}\right)$. The reconstruction $\hat{S}_{0}\left[f\left(\omega_{x}, \omega_{y}\right)\right]$ from a dyadic wavelet decomposition can be represented recursively as

$$
\begin{aligned}
\hat{S}_{j}\left[f\left(\omega_{x}, \omega_{y}\right)\right]= & \hat{W}_{j+1}^{1}\left[f\left(\omega_{x}, \omega_{y}\right)\right] K\left(2^{j} \omega_{x}\right) L\left(2^{j} \omega_{y}\right) \\
& +\hat{W}_{j+1}^{2}\left[f\left(\omega_{x}, \omega_{y}\right)\right] L\left(2^{j} \omega_{x}\right) K\left(2^{j} \omega_{y}\right) \\
& +\hat{S}_{j+1}\left[f\left(\omega_{x}, \omega_{y}\right)\right] \bar{H}\left(2^{j} \omega_{x}\right) \bar{H}\left(2^{j} \omega_{y}\right)
\end{aligned}
$$

where $\bar{H}$ is the complex conjugate of $H$. The DWT decomposition and reconstruction based on the above recursive relations are shown as a block diagram in Fig. 1. The reconstructed $f^{*}(m, n)$ is equal to $f(m, n)$ when no processing is performed on $\mathcal{W}[f(m, n)]$. The finite impulse response (FIR) filters shown in Table I were defined in [23], similar to those 
TABLE I

IMPULSE Responses of Filters $H(\omega), G(\omega), K(\omega)$, AND $L(\omega)$

FIR filters

\begin{tabular}{rlccl}
$n$ & $h(n)$ & $g(n)$ & $k(n)$ & \multicolumn{1}{c}{$l(n)$} \\
\hline-4 & & & & 0.001953125 \\
-3 & & & -0.00390625 & 0.015625 \\
-2 & 0.0625 & & -0.03515625 & 0.0546875 \\
-1 & 0.25 & 1.0 & -0.14453125 & 0.109375 \\
0 & 0.375 & -1.0 & -0.36328125 & 0.63671875 \\
1 & 0.25 & & 0.36328125 & 0.109375 \\
2 & 0.0625 & & 0.14453125 & 0.0546875 \\
3 & & & 0.03515625 & 0.015625 \\
4 & & & 0.00390625 & 0.001953125 \\
\hline
\end{tabular}

developed in [24]. The discrete function $H(\omega)$ is defined as $\Sigma_{n} h(n) e^{-i \omega n}$. Our speckle reduction and contrast enhancement were accomplished in the transform domain by judicious multiscale nonlinear processing of such wavelet coefficients obtained in this manner $\left(W_{j}^{d}[f(m, n)]\right)_{d=1,2,1 \leq j \leq J}$.

\section{SPECKLE REduction AND CONTRAST ENHANCEMENT}

Ultrasound imaging techniques are widely used in medical diagnosis. Its noninvasive nature, low cost, portability, and real-time image formation make ultrasound imaging an attractive means for medical diagnosis, especially in cardiology. One of the limitations of ultrasound images is poor image quality affected by speckle noise. Speckle reduction remains a difficult problem due to the lack of reliable models to estimate noise. Speckle under different imaging media, such as laser, radar, or ultrasound, may appear distinct. However, the granular pattern under each of these media is produced by coherent interference related to the roughness of object surfaces. An approximate speckle noise model [1] is formulated here without temporal averaging. We apply this speckle noise model within a framework of multiscale wavelet analysis for speckle reduction and feature enhancement of echocardiographic images. By incorporating a feature enhancement mechanism into a denoising process, we are able to not only reduce noise, but also restore features of importance to cardiology. Since in this paper we are more interested in noise reduction and feature enhancement of images, the problems of denoising and enhancement are formulated directly in two dimensions.

\section{A. Approximate Speckle Noise Model}

An accurate and reliable model of speckle noise is desirable for efficient speckle reduction. It remains a difficult problem. Jain [1] presented a general model for speckle noise as

$$
f(x, y)=g(x, y) \eta_{m}(x, y)+\eta_{a}(x, y)
$$

where $g(x, y)$ is an unknown 2-D function, such as a noise-free original image, to be recovered, $f(x, y)$ is a noisy observation of $g(x, y), \eta_{m}(x, y)$ and $\eta_{a}(x, y)$ are multiplicative and additive noise respectively, $x$ and $y$ are variables of spatial locations, $(x, y) \in \boldsymbol{R}^{2}$. Since the effect of additive noise (such as sensor noise) is considerably small compared to that of multiplicative noise (coherent interfering) $\left(\left\|\eta_{a}(x, y)\right\|^{2} \ll\left\|g(x, y) \eta_{m}(x, y)\right\|^{2}\right)$ in echocardiograms,
(8) can be approximated by

$$
f(x, y)=g(x, y) \eta_{m}(x, y) .
$$

To separate the noise from the original image, we take a logarithmic transform on the both sides of (9)

$$
\log (f(x, y))=\log (g(x, y))+\log \left(\eta_{m}(x, y)\right) .
$$

Equation (10) can also be rewritten as

$$
f^{l}(x, y)=g^{l}(x, y)+\eta_{a}^{l}(x, y) .
$$

Now we can approximate $\eta_{a}^{l}(x, y)$ as additive white noise and may apply various wavelet-based approaches for additive noise reduction. With uniform sampling, we obtain the discrete version of (11) as

$$
f^{l}(m, n)=g^{l}(m, n)+\eta_{a}^{l}(m, n)
$$

where $(m, n) \in Z^{2}, f^{l}(m, n) \equiv f^{l}\left(m T_{x}+s_{x}, n T_{y}+s_{y}\right)$, $T_{x}$ and $T_{y}$ are sampling periods along horizontal and vertical directions, $s_{x}$ and $s_{y}$ are sampling shifts. A DWT is a linear transform, so we have

$$
\mathcal{W}\left[f^{l}(m, n)\right]=\mathcal{W}\left[g^{l}(m, n)\right]+\mathcal{W}\left[\eta_{a}^{l}(m, n)\right]
$$

where

$$
\begin{gathered}
\mathcal{W}\left[g^{l}(m, n)\right]=\left\{\left(W_{j}^{d}\left[g^{l}(m, n)\right]\right)_{d=1,2,1 \leq j \leq J},\right. \\
\left.S_{J}\left[g^{l}(m, n)\right]\right\} \\
\mathcal{W}\left[\eta_{a}^{l}(m, n)\right]=\left\{\left(W_{j}^{d}\left[\eta_{a}^{l}(m, n)\right]\right)_{d=1,2,1 \leq j \leq J}\right. \\
\left.S_{J}\left[\eta_{a}^{l}(m, n)\right]\right\} .
\end{gathered}
$$

Since speckle noise lies in high spatial frequency, it will reduce to near zero after a finite number of repeated smoothing operations, so $S_{J}\left[\eta_{a}^{l}(m, n)\right] \rightarrow 0$. In fact, at most a five-level wavelet decomposition is needed to smooth out noise for most noise reduction applications we conducted. This is why we carry out speckle noise reduction through eliminating noise energy $\Sigma_{d, j}\left(W_{j}^{d}\left[\eta_{a}^{l}(m, n)\right]\right)^{2}$. For image restoration purposes, it is desirable to recover $\mathcal{W}\left[g^{l}(m, n)\right]$ from a DWT $\mathcal{W}\left[f^{l}(m, n)\right]$ by reducing $\mathcal{W}\left[\eta_{a}^{l}(m, n)\right]$ in the wavelet domain. For noise reduction and feature enhancement, we want to further increase the sharpness of features of interest, such as myocardial boundaries, through nonlinear stretching via feature energy gain on signal details $W_{j}^{d}\left[g^{l}(m, n)\right]$.

Jain showed a similar homomorphic approach [1, pp. 313-316] for speckle reduction of images with undeformable objects through temporal averaging and homomorphic Wiener filtering. The motion and deformable nature of human hearts through time prevents us from getting the same status of the left ventricle for multiple frames. Since we treated noise and features differently, we were able to accomplish a better result than denoising only algorithms.

\section{B. Wavelet Shrinkage and Feature Emphasis}

During the last few years, a number of wavelet-based denoising techniques [3], [4], [8], [22], [25], [26] were developed while several wavelet-based contrast enhancement methods [6], [7], [17] were available. To achieve both objectives of denoising and enhancement, we need 1) a representation which can separate features from noise, 2) effective denoising and feature enhancement techniques. Wavelet shrinkage methods, 
such as hard thresholding and Donoho's soft thresholding, have been investigated for speckle reduction of images on a logarithmic scale. An advantage of soft thresholding is that it provides smoothness while hard thresholding preserves features. After converting multiplicative noise into additive noise [see (10)] through a log homomorphic transform, we apply soft thresholding at fine scales (such as levels 1 and/or 2) and hard thresholding within middle levels (such as levels 3 and/or 4) to eliminate noise. We also applied a method of nonlinear contrast stretching in a wavelet domain to enhance myocardial features. In our approach, we take advantage of both thresholding methods. Donoho's soft thresholding method [26] was developed on an orthonormal wavelet transform [27], primarily with Daubechies's Symmlet 8 basis wavelet. Previous denoising results have shown some undesirable side effects, including pseudo-Gibbs phenomena [8]. By employing a DWT and an antisymmetric basis wavelet with few oscillations, we are relatively free from such side effects after denoising. Our experiments show that a DWT with a firstorder derivative of a smoothing function as its basis wavelet can separate feature energy and noise energy in the transform domain. In this algorithm, we adopt regularized soft thresholding (wavelet shrinkage) to remove noise energy within the finer scales and nonlinear processing of feature energy to enhance contrast. Hard thresholding is incorporated for preserving features while removing small noise perturbations within the middle levels of analysis. The wavelet shrinkage and feature emphasis techniques were described in [10], which are refined and included here for the completeness of the algorithm.

1) Wavelet Shrinkage by Soft Thresholding: Soft thresholding [26] operation can be represented as

$$
u=\mathcal{T}(v, t)=\operatorname{sign}(v)(|v|-t)_{+}
$$

where the threshold parameter $t$ is proportional to the noise level $\sigma$, and $u$ is the result of soft thresholding and has the same sign as $v$ if nonzero. Expression $(|v|-t)_{+}$is defined as

$$
(|v|-t)_{+}= \begin{cases}|v|-t, & \text { if }|v|>t \\ 0, & \text { otherwise }\end{cases}
$$

DWT coefficients can be modified for noise reduction by

$$
W_{j}^{d, *}[f(m, n)]=\mathcal{T}\left(W_{j}^{d}[f(m, n)], t_{j}^{d}\right)
$$

where $d=1,2, j=1, \cdots, k, k \leq J$, and $t_{j}^{d}$ is, in general, a threshold related to the noise level, orientation, and scale. Donoho's method of soft thresholding uses a single global threshold. Since noise coefficients under a DWT have average decay through fine-to-coarse scales, we regulate soft thresholding by applying coefficient dependent thresholds at distinct scales. Fig. 2 shows a soft thresholding operation compared with hard thresholding.

The regulated threshold $t_{j}^{d}$ can be computed through a linearly decreasing function

$$
t_{j}^{d}= \begin{cases}\left(T_{\max }-\alpha(j-1)\right) \sigma_{j}^{d}, & \text { if } T_{\max }-\alpha(j-1)>T_{\min }, \\ T_{\min } \sigma_{j}^{d}, & \text { otherwise }\end{cases}
$$

where $\sigma_{j}^{d}$ is the standard deviation, $\alpha$ is a decreasing factor between two consecutive levels, $T_{\max }$ is a maximum factor

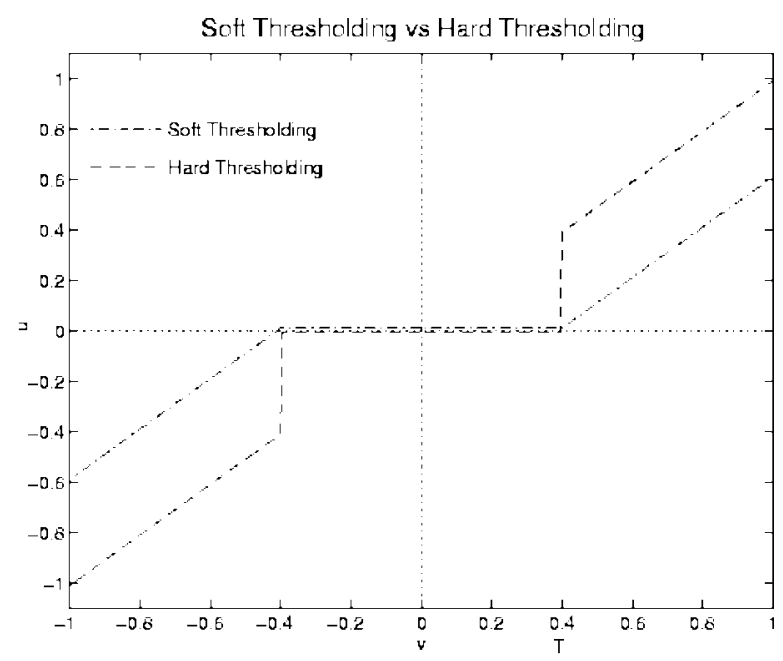

Fig. 2. Thresholding methods for denoising, where $v(x)$ is an input to thresholding operators and $u(x)$ is the output.

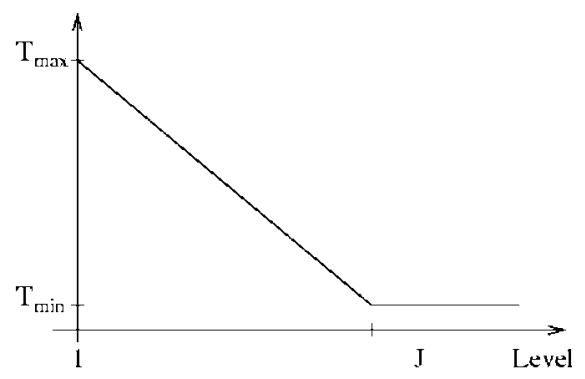

Fig. 3. A simple scaling factor function.

for $\sigma_{j}^{d}$ while $T_{\min }$ is a minimum factor, $1 \leq j \leq J$, and $d \in\{1,2\}$. For the case of an unknown noise level, we use $\sigma_{j}^{d}$ to estimate the noise level of a signal or image. Threshold $t_{j}^{d}$ is primarily calculated using $\sigma_{j}^{d}$ and a decreasing factor $T_{\max }-\alpha(j-1)$. Fig. 3 shows one simple scaling factor function for the computation of regulated thresholds.

2) Feature Emphasis by Generalized Adaptive Gain: Adaptive gain nonlinear processing [7], [17] has been successfully utilized to enhance features in digital mammography. The adaptive gain operation was generalized to incorporate hard thresholding to avoid amplifying noise and remove small noise perturbations within middle scales [10]. A generalized adaptive gain (GAG) operator is defined as

$$
E_{\mathrm{GAG}}(v)= \begin{cases}0, & \text { if }|v|<T_{1}, \\ \operatorname{sign}(v) T_{2}+\bar{u}, & \text { if } T_{2} \leq|v| \leq T_{3}, \\ v, & \text { otherwise }\end{cases}
$$

where $\bar{u}=a\left(T_{3}-T_{2}\right)(\operatorname{sigm}(c(u-b))-\operatorname{sigm}(-c(u+b))), v \in$ $[-1,1], u=\operatorname{sign}(v)\left(|v|-T_{2}\right) /\left(T_{3}-T_{2}\right), b \in(0,1), 0 \leq T_{1} \leq$ $T_{2}<T_{3} \leq 1, c$ is a gain factor, and $a$ can be computed as

$$
\begin{aligned}
a & =\frac{1}{\operatorname{sigm}(c(1-b))-\operatorname{sigm}(-c(1+b))} \\
\operatorname{sigm}(x) & =\frac{1}{1+e^{-x}} .
\end{aligned}
$$




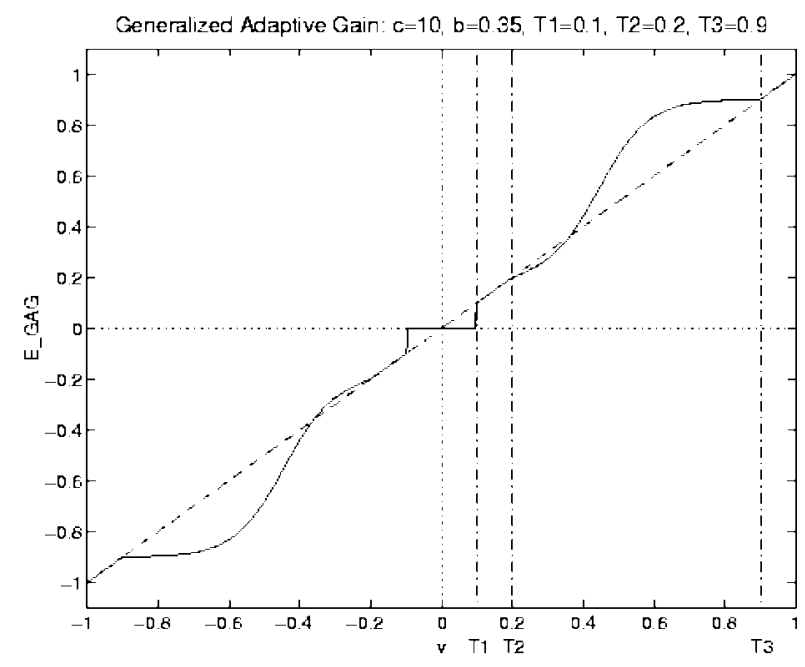

Fig. 4. A sample GAG function.

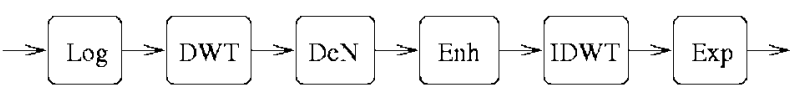

Fig. 5. An algorithm for speckle reduction and contrast enhancement.

$E_{\mathrm{GAG}}(v)$ is simply an enhancement operator, and $T_{1}, T_{2}$, and $T_{3}$ are selected parameters. When $T_{1}=T_{2}=0$ and $T_{3}=1$, the expression is equivalent to an adaptive gain operator in [17]. The interval $\left[T_{2}, T_{3}\right]$ serves as a sliding window for feature selectivity. It can be adjusted to emphasize features within a specific range of variation. The GAG operator is used to accomplish contrast enhancement through nonlinear stretching of wavelet coefficients. By selecting a gain, a window, and other parameters, we achieve distinct enhancement. Thus, through this nonlinear operator, DWT coefficients are processed for feature enhancement by

$$
\begin{aligned}
W_{j}^{d, *}[f(m, n)] & =M_{j}^{d} E_{\mathrm{GAG}}\left(W_{j}^{d}[f(m, n)] / M_{j}^{d}\right) \\
M_{j}^{d} & =\max _{m, n}\left(\left|W_{j}^{d}[f(m, n)]\right|\right)
\end{aligned}
$$

where position $(m, n) \in D$, the domain of $f(m, n), d=1,2$, $j \in\{k, \cdots, J\}$, and $1 \leq k \leq J$. Fig. 4 presents an adaptive gain function with feature selectivity.

3) Overview of Algorithm for Speckle Reduction and Feature Enhancement: The functionality and operations of the algorithm can be approximated by the formula

$$
\left(g^{*}(m, n)\right)=\left(\exp \left(\mathcal{W}^{-1}\left[E_{\mathrm{GAG}}^{s}\left(\mathcal{T}_{s}(\mathcal{W}[\log (f(m, n))])\right)\right]\right)\right)
$$

where $(m, n) \in Z^{2}$. For the denoising operator $\mathcal{T}_{s}$ and enhancement operator $E_{\mathrm{GAG}}^{s}$, we have added the subscript and superscript $s$ to show the selectivity of various parameters in the two operations. In $(22), g^{*}(m, n)$ is a recovered image of an unknown $g(m, n)$ defined by (8) with features enhanced. The complete algorithm with six major steps is shown as a block diagram in Fig. 5.

Our test images included noise-induced spikes among wavelet coefficients at finer scales due to a significant amount of intensity drop caused by signal cancellation under coherent interference. We incorporated a spatially weighted averaging

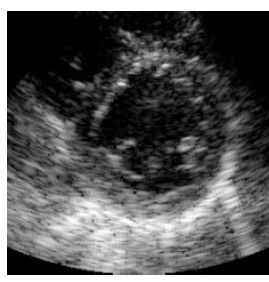

(a)

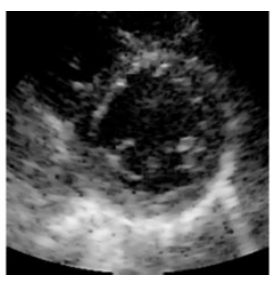

(b)

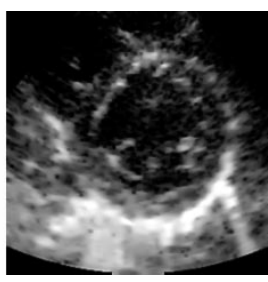

(c)
Fig. 6. Results of denoising and enhancement. (a) A noisy ED frame. (b) Wavelet shrinkage denoising-only method. (c) DWT-based denoising and enhancement.

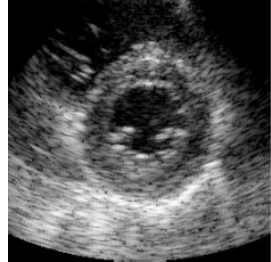

(a)

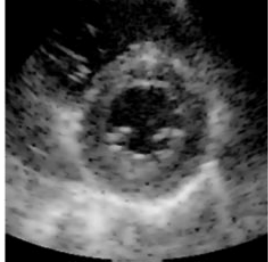

(b)

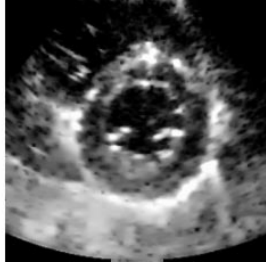

(c)
Fig. 7. Results of denoising and enhancement. (a) A noisy ES frame. (b) Wavelet shrinkage denoising-only method. (c) DWT-based denoising and enhancement.

operation during denoising to further diffuse the pulse energy within one or two octaves of high frequency. For instance, we used a $3 \times 3$ window to smooth wavelet coefficients at the first level before applying wavelet shrinkage to clinical image processing under Section V. The center pixel had weight four, the four horizontal and vertical neighbor pixels had weight two, and the four diagonal neighbor pixels had weight of one.

\section{EXPERIMENTAL RESULTS AND DISCUSSIONS}

Our multiscale homomorphic algorithm for speckle reduction and feature enhancement was tested on echocardiograms of varying quality. These image sequences were acquired from the parasternal short-axis view. Figs. 6 and 7 present the results of denoising with or without feature enhancement on end diastolic (ED) and end systolic (ES) frames. The speckled original frames are shown first. Results from wavelet shrinkage denoising only and denoising with enhancement are shown in the Fig. 6(b) and (c), respectively. Fig. 8 shows a nonlinear operator for enhancing the image in Fig. 7(a). This operator looks much different from Fig. 4 because of the $\log$ transform. Experimental results are also compared with other speckle reduction techniques, such as median filtering, extreme sharpening combined with median filtering [18], [19], homomorphic Wiener filtering, and a wavelet shrinkage denoising [25], [26]. Figs. 9 and 10 show sample results of the above mentioned methods on two typical frames from two different echocardiographic sequences with the left ventricle as the region of interest. Since the noise-free original image was not available for our test image, the homomorphic Wiener filtering method used the Gaussian low-pass filtered version (with $\mu=0$ and $\sigma=1.1$ ) as an approximation of the original image and applied Wiener smoothing filter [1, pp. 280-281] to reduce speckle from the noisy image. Fig. 9(a) is a sample noisy image. The result of median filtering with a $5 \times 5$ mask 


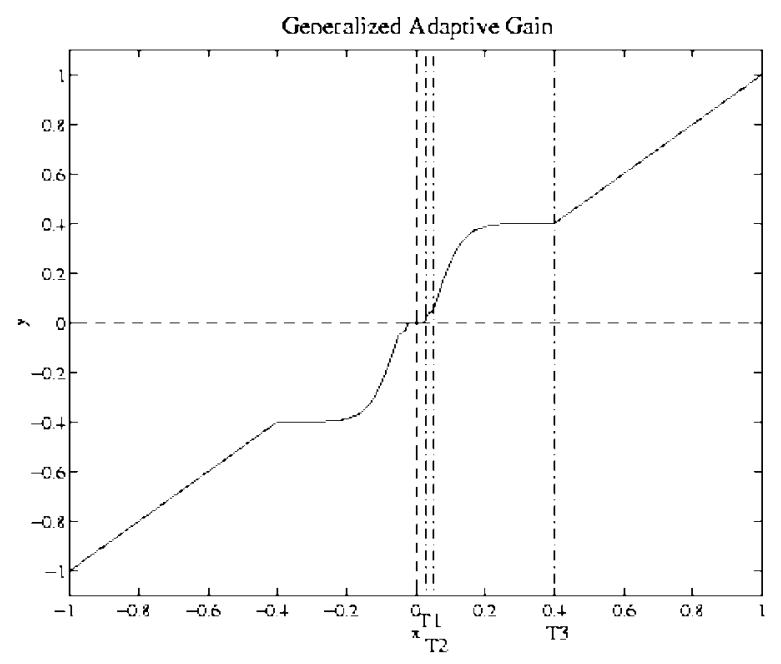

Fig. 8. A GAG function for processing an ECG in Fig. 7(a). The parameter setting are $b=0.08, c=10.0, T 1=0.03, T 2=0.05$, and $T 3=0.4$.

is shown in Fig. 9(b). Fig. 9(c) displays a sample result of extreme sharpening combined with median filtering. The result from homomorphic Wiener filtering is shown in Fig. 9(d). The last image, Fig. 9(e) and (f), displays the result from wavelet shrinkage denoising only and our denoising and enhancement algorithm. The setting of parameters for Fig. 9(e) are as follows. Wavelet shrinkage denoising was applied only at the first and second levels with the scaling factor in (16) being 0.4 and 0.2 , respectively. Fig. 9(e) shows the result of denoising. Fig. 9(f) resulted from the same denoising parameters plus a feature enhancement operation before reconstruction. The feature enhancement operation was performed only at the third and fourth levels. The parameters were $B=0.08, C=10$, $T 1=0, T 2=0.05$, and $T 3=0.35$. In this experiment, we used relatively large thresholds for wavelet shrinkage compared to those used in the next experiment. More noise has been removed, therefore, we set $T 1=0$, so that there would be no further reduction in noise during feature enhancement. We can fine tune the thresholds and $T 1$ to optimize the noise reduction and feature preservation. The parameter setting for Fig. 10(e) are as follows. We have also applied wavelet shrinkage denoising at the first and second levels with the scaling factor in (16) being 0.35 and 0.15 , respectively. Fig. 10(e) shows the result of the denoising only method. Fig. 10(f) had the same denoising parameters plus feature enhancement. The parameters for feature enhancement at the third and fourth levels are $B=0.086, C=7, T 1=0.02$, $T 2=0.04$, and $T 3=0.35$. The algorithm produces smoothness inside a uniform region and contrast along structure and object boundaries in addition to speckle reduction. The denoised and enhanced results of noisy echocardiographic images from this algorithm appear to outperform the results from soft thresholding denoising alone. Our current algorithm is implemented such that most parameters are set dynamically for adaptive denoising and feature enhancement.

The parameters used in the algorithm, including those shown in Fig. 8, were adjusted by trial-and-error. Through our study and experiments, we suggest that the following guidelines will

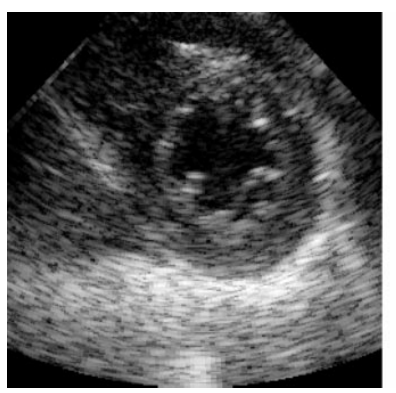

(a)

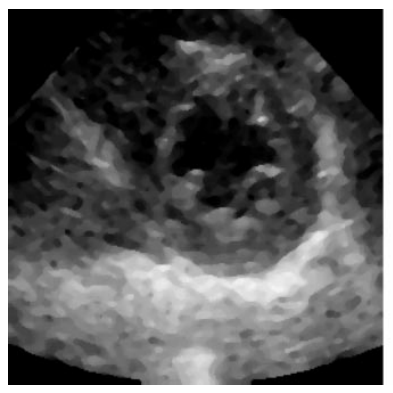

(c)

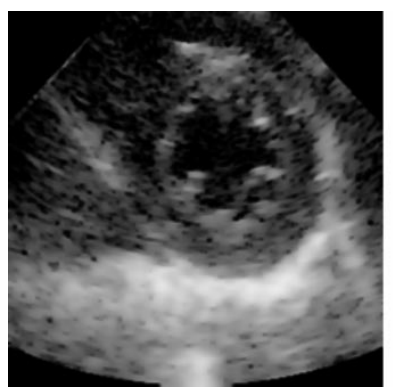

(e)

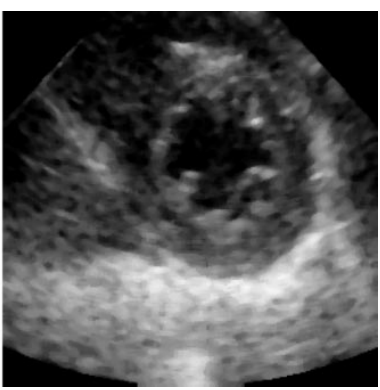

(b)

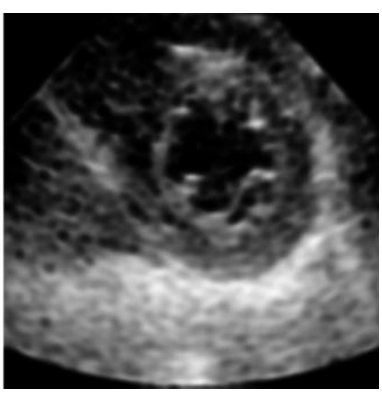

(d)

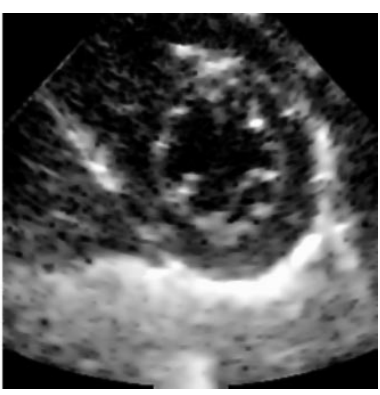

(f)
Fig. 9. Results of various denoising methods. (a) Original image with speckle noise. (b) Median filtering. (c) Extreme sharpening combined with median filtering. (d) Homomorphic Wiener filtering. (e) Wavelet shrinkage denoising only. (f) DWT-based denoising with enhancement.

help to fine tune these parameters and to produce enhancement results. To enhance fine signal details, such as microcalcifications in mammographic images, we try to modify wavelet coefficients at the first three levels [28]. This is because edges with small sizes can be characterized by relatively large wavelet coefficients at the first few levels. To avoid amplifying digitization noise, we add less energy gain to wavelet coefficients at the first level than those at the second and third levels. For enhancing a large structure boundary, such as myocardial borders, we add gain primarily to coefficients at the third and fourth levels. As expected, increasing signal energy at the fine scales enhanced high-frequency features such as sharpened step edges, while increasing signal energy at the coarse scales improved the visibility of large structures and object boundaries. The histogram and energy distribution of wavelet coefficients at each level and orientation can provide helpful information for identifying the distribution of noise and feature coefficients, and we can adjust all related parameters accordingly.

The settings of $T_{1}, T_{2}$, and $T_{3}\left(0 \leq T_{1} \leq T_{2}<T_{3} \leq 1\right)$ are as follows. In a noisy image, coefficients below $T_{1} * M_{j}^{d}$ are more likely attributed to noise, where $M_{j}^{d}$ is the maximum 


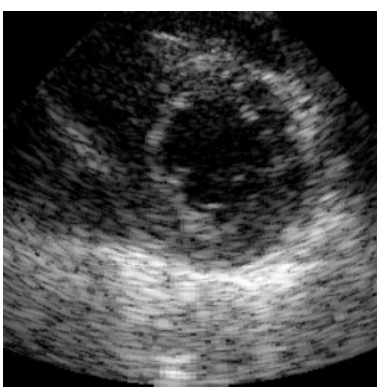

(a)

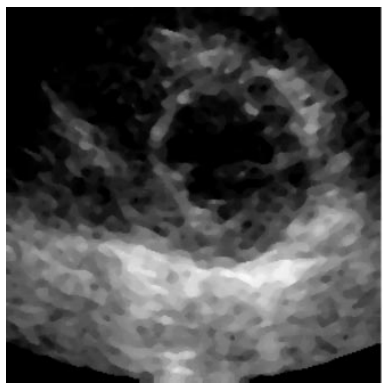

(c)

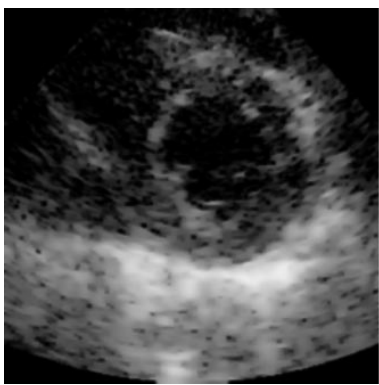

(e)

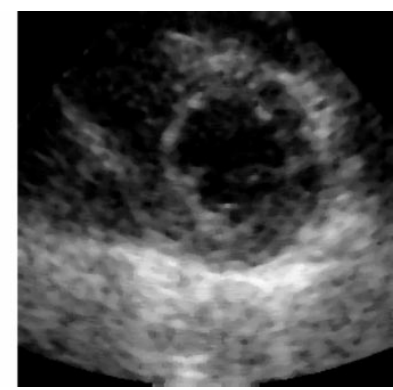

(b)

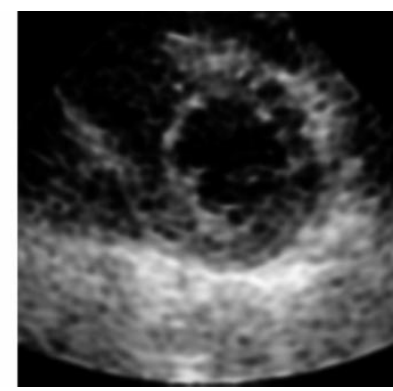

(d)

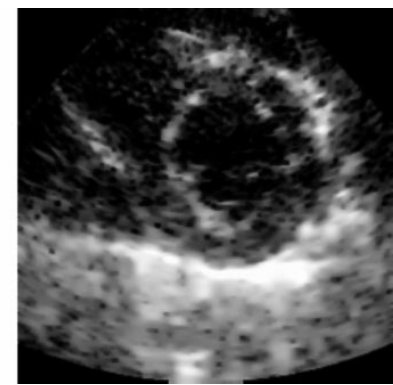

(f)
Fig. 10. Results of various denoising methods. (a) Original image with speckle noise. (b) Median filtering. (c) Extreme sharpening combined with median filtering. (d) Homomorphic Wiener filtering. (e) Wavelet shrinkage denoising-only method. (f) DWT-based denoising and enhancement.

coefficient at level $j$ and orientation $d$ (horizontal or vertical). These coefficients are set to zero to suppress noise. For an image with a high level of noise, $T_{1}$ can be set relatively large. The parameter $T_{1}$ can be set close to zero for a relatively noisefree image. The coefficients in the range of $\left[T_{1} * M_{j}^{d}, T_{2} * M_{j}^{d}\right]$ are considered at risk for enhancement because they can represent noise or weak feature coefficients with a relatively equal chance. They are left unchanged to avoid amplifying noise. The coefficients in the range of $\left[T_{2} * M_{j}^{d}, T_{3} * M_{j}^{d}\right]$ are really what we want to enhance by adding gain. The contrast of a feature with coefficients larger than $T_{3} * M_{j}^{d}$ is already good, so we leave those coefficients unchanged. We can adjust $T_{2}$ and $T_{3}$ toward zero to enhance weak features with coefficients in the range of $\left[T_{2} * M_{j}^{d}, T_{3} * M_{j}^{d}\right]$. A large window $\left(\left[T_{2} * M_{j}^{d}, T_{3} * M_{j}^{d}\right]\right)$ helps to achieve the overall enhancement while a small window can be used for selected enhancement. An enhancement function with a large $c$ in (17) adds more gain to selected wavelet coefficients and the parameter can be adjusted to achieve a distinct effect of feature enhancement. Parameter $b$ can be used to control the shape of an enhancement function.

\section{Clinical Case Study}

A study of clinical images was conducted to investigate the effect of denoising on the consistency and reliability to manually defined borders of the left ventricle in 2-D short-axis echocardiographic images by expert observers. Experimental results indicate the algorithm is promising. Borders defined by experts exhibit less variation after processing. It seems that in echocardiograms, where no real borders are clearly visible and are often incomplete, expert borders usually indicate a close range where real borders may occur. When two expert borders agree with each other, the range of real borders is more likely limited around the two expert borders. The study of clinical images shows that denoising and feature enhancement help the consistency and reliability of manually defined borders by expert observers.

The set of test images included in our study of clinical images was selected from an echocardiographic database exhibiting diverse image quality. Sixty systolic sequences of 2-D short-axis echocardiographic images were selected. Half of the test images were rated as good quality while the rest were considered as poor quality. For more details about how these echocardiographic sequences were acquired, see reference [29]. Statistical results have shown that there is some improvement in consistency and reliability for manually defined borders by expert observers examining denoised images compared to their original noisy images. Quantitative measurements were calculated in terms of the mean of absolute border differences (MDistDiff) in distance $(\mathrm{mm})$ and the mean of border area differences (MAreaDiff) in $\mathrm{cm}^{2}$. The border difference was measured by its close approximation in 64 radial directional difference from an estimated center [29] of the left ventricle. MAreaDiff is defined as the absolute area difference of two borders. Manually defined borders by experts looking at poor images were improved after denoising. The statistical results of quantitative measurements of two sets of borders manually defined by two experts are shown in Table II. The statistical computation results listed under the column "Ori" are the quantitative measurements between two sets of expert borders on the original speckled images while the results under the column "Enh" are based on the denoised and enhanced images. It is worth mentioning that a single set of denoising and enhancement parameters was used to process all the test echocardiographic images used in this study. We suggest that a single value set of parameters should suffice for denoising and enhancing a class of images with a similar noise pattern and selected features. For the clinical images of this study, wavelet shrinkage denoising was applied at the first and second levels with the scaling factors in (21) being 0.15 and 0.12 , respectively. The parameters for feature enhancement at the third and fourth levels are $B=0.01, T 1=0.05, T 2=0.05$, and $T 3=0.55$. The values of $\mathrm{C}$ at level 3 and 4 are 8 and 40, respectively. We used relatively small thresholds to avoid over-smoothing the images and added more gain at level 4 than level 3 .

Fig. 11 shows the correlation between the areas delineated by the two expert observers. The four diagrams in Fig. 11(a) present the correlation of ED Epi (epicardial) border areas, 
TABLE II

Quantitative Measurements of Manually Defined Borders

\begin{tabular}{|c|c|c|c|c|c|c|c|}
\hline \multirow{3}{*}{$\begin{array}{l}\text { MDistDiff } \\
\text { (in mm) }\end{array}$} & \multirow{3}{*}{$\begin{array}{l}\text { Endo } \\
\text { Epi }\end{array}$} & \multicolumn{2}{|c|}{$\begin{array}{l}\text { All Images } \\
\text { Ori versus Enh }\end{array}$} & \multicolumn{2}{|c|}{$\begin{array}{l}\text { Good Images } \\
\text { Ori versus Enh }\end{array}$} & \multicolumn{2}{|c|}{$\begin{array}{l}\text { Poor Images } \\
\text { Ori versus Enh }\end{array}$} \\
\hline & & 2.1040 & 1.8168 & 1.5972 & 1.5322 & 2.6118 & 2.1014 \\
\hline & & 1.7846 & 1.6743 & 1.3979 & 1.5886 & 2.1713 & 1.7601 \\
\hline MAreaDiff & Endo & 2.3731 & 1.8893 & 1.6597 & 1.4543 & 3.0865 & 2.2058 \\
\hline (in $\mathrm{cm}^{2}$ ) & Epi & 2.5676 & 2.0799 & 1.5823 & 1.9540 & 3.5528 & 2.324 \\
\hline
\end{tabular}
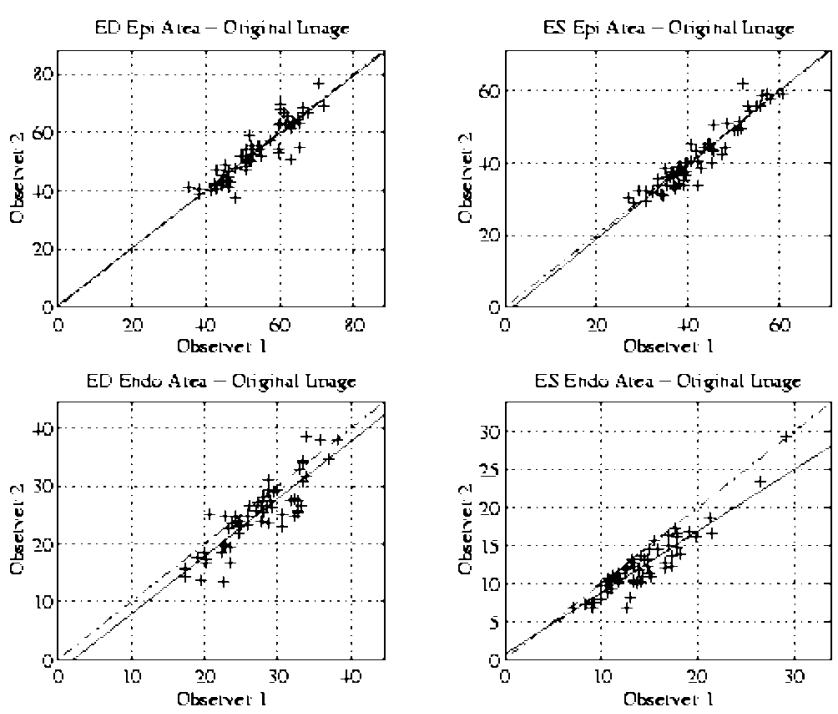

(a)
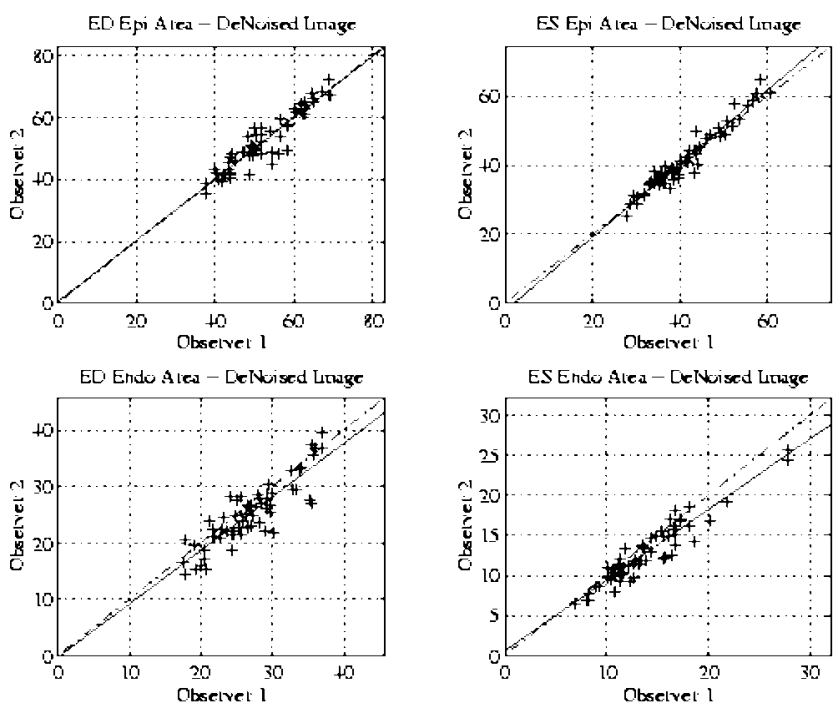

(b)

Fig. 11. Area correlation between manually defined borders by two expert cardiologist observers: (a) on the original noisy images and (b) on the denoised images with features restored or enhanced.

ES Epi border areas, ED Endo (endocardial) border areas, and ES Endo border areas on the original noisy images. The four diagrams in Fig. 11(b) show similar results for the denoised images with features restored or enhanced. The solid lines in the figure are the linear regression lines, while the dash and dotted lines are ideal regression lines. From the diagrams, it is clear that the points which represent the two expert border areas on the same denoised image are, in general, more toward

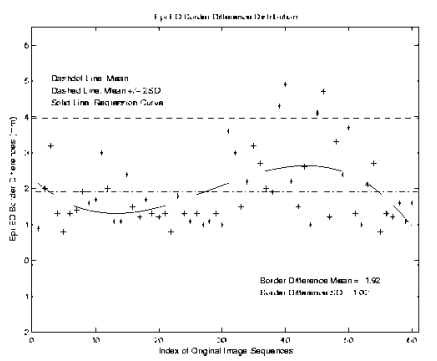

(a)

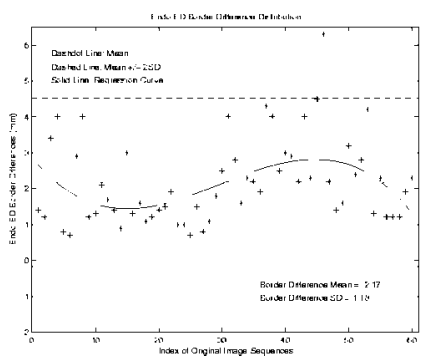

(c)

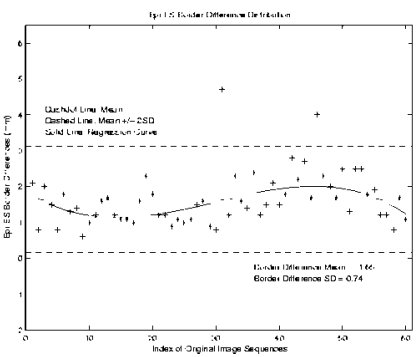

(b)

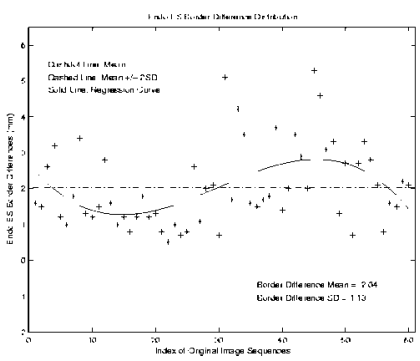

(d)
Fig. 12. Border difference variation on the original images. (a) Distribution of Epi ED border differences. (b) Distribution of Epi ES border differences. (c) Distribution of Endo ED border differences. (d) Distribution of Endo ES border differences. The solid lines are the third-order polynomial fitting curves.

the ideal regression line. Additional improvement can be seen on the Endo area correlation for the denoised images. In most echocardiograms, there is usually less Endo border information than Epi border information. Noisy border information affects border interpolation by human observers for the manually defined borders. After denoising, Endo border information is improved, so the expert border areas tend to agree with each other, especially ES Endo areas. The statistical computation results shown in Table II, support this analysis.

Fig. 12 shows the distributions of mean border differences on the original images; 1 ) the distribution of Epi ED border differences, 2) the distribution of Epi ES border differences, 3) the distribution of Endo ED border differences, and 4) the distribution of Endo ES border differences. A mean border difference was computed along 64 equiangular radial segments from a common center [29]. Each of the line segments intersects the two manual borders, each once. The absolute difference between the two intersection points along each segment was computed before averaging. Fig. 13(a)-(d) shows the distributions of mean border differences on the enhanced images, similar to Fig. 12(a)-(d). The solid lines in Figs. 12 and 13 are the third-order polynomial fitting curves in a least-squares sense. With the same scale for both Figs. 12 and 13, Fig. 13 shows that border distance differences for enhanced images have smaller means and standard deviations than the corresponding 


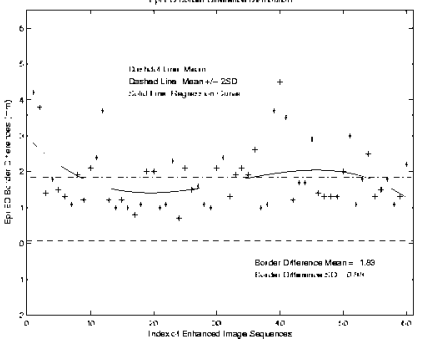

(a)

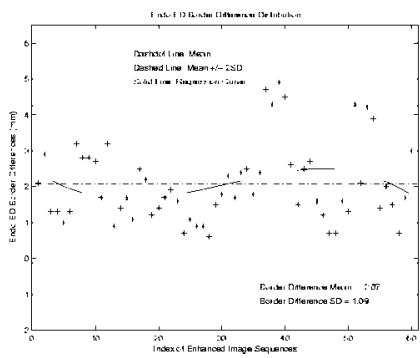

(c)

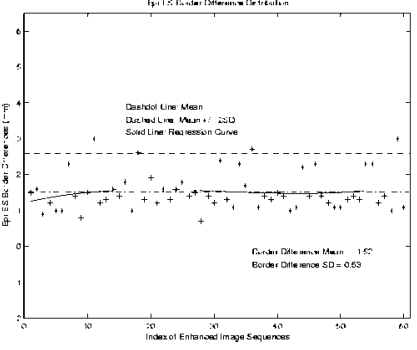

(b)

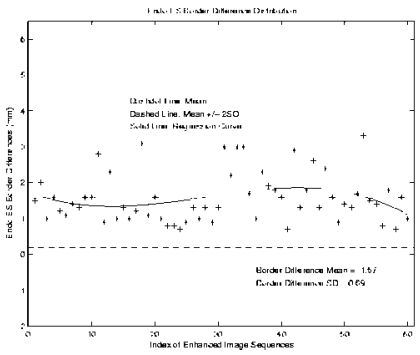

(d)
Fig. 13. Border difference variation on the enhanced images. (a) Distribution of Epi ED border differences. (b) Distribution of Epi ES border differences. (c) Distribution of Endo ED border differences. (d) Distribution of Endo ES border differences. The solid lines are the third-order polynomial fitting curves.

differences for the original noisy images as shown in Fig. 12. For more details about this study, we refer the reader to [30].

\section{CONCLUSIONS}

In this paper, we presented a multiscale homomorphic approach for speckle reduction and feature enhancement. We showed that two conflicting objectives of denoising and enhancement can be achieved through multiscale nonlinear processing. Through a fine-to-coarse scale space analysis of a speckled image on a logarithmic scale, distinct behaviors of noise and features can be differentiated. We took advantage of both soft thresholding and hard thresholding wavelet shrinkage techniques. Nonlinear stretching of wavelet coefficients was subsequently performed for feature restoration and enhancement. The algorithm was tested by applying it to a variety of ultrasound images from an echocardiographic database exhibiting diverse image quality. Subjective image quality was improved. The statistical results from a study of clinical images show overall improvement in the consistency of borders manually defined by expert observers after denoising and enhancement.

\section{REFERENCES}

[1] A. K. Jain, Fundamentals of Digital Image Processing. Englewood Cliffs, NJ: Prentice-Hall, 1989.

[2] E. A. Geiser, D. C. Wilson, G. L. Gibby, J. Billett, and D. A. Conetta, "A method for evaluation of enhancement operations in two-dimensional echocardiographic images," J. Amer. Soc. Echocardiogr., vol. 4, no. 3, pp. 235-246, May 1991.

[3] J. Lu, J. B. Weaver, D. M. Healy Jr., and Y. Xu, "Noise reduction with multiscale edge representation and perceptual criteria," in Proc. IEEESP Int. Symp. Time-Frequency and Time-Scale Analysis, Victoria, B.C., 1992, pp. 555-558.

[4] R. R. Coifman and F. Majid, "Adapted waveform analysis and denoising," in Progress in Wavelet Analysis and Applications, Y. Meyer and S.
Roques, Eds., Editions Frontiers. Gif-sur-Yvette Cedex, France, 1993, pp. 63-76.

[5] H. Guo, J. E. Odegard, M. Lang, R. A. Gopinath, I. W. Selesnick, and C. S. Burrus, "Wavelet based speckle reduction with application to SAR based ATD/R," in Proc. ICIP, 1994, vol. 1, pp. 75-79.

[6] J. Lu and D. M. Healy, Jr., "Contrast enhancement of medical images using multiscale edge representation," in Wavelet Applications, Proc. SPIE, Orlando, FL, 1996, vol. 2242, pp. 711-719.

[7] A. F. Laine, S. Schuler, J. Fan, and W. Huda, "Mammographic feature enhancement by multiscale analysis," IEEE Trans. Med. Imag., vol. 13, no. 4, pp. 725-740, 1994.

[8] R. R. Coifman and D. L. Donoho, "Translation-invariant denoising," in Wavelets and Statistics, A. Antoniadis and G. Oppenheim, Eds. New York: Springer-Verlag, 1995, pp. 125-150.

[9] J. Fan and A. Laine, "Multiscale contrast enhancement and denoising in digital radiographs," in Wavelets in Medicine and Biology, A. Aldroubi and M. Unser, Eds. Boca Raton, FL: CRC, 1996, pp. 163-189.

[10] X. Zong, E. A. Geiser, A. F. Laine, and D. C. Wilson, "Homomorphic wavelet shrinkage and feature emphasis for speckle reduction and enhancement of echocardiographic images," in Medical Imaging: Image Processing, Proc. SPIE, 1996, vol. 2710, pp. 658-667.

[11] V. Chalana, D. T. Linker, D. R. Haynor, and Y. Kim, "A multiple active contour model for cardiac boundary detection on echocardiographic sequences," IEEE Trans. Med. Imag., vol. 15, pp. 290-298, June 1996.

[12] J. W. Goodman, "Some fundamental properties of speckle," J. Opt. Soc. Amer., vol. 66, no. 11, pp. 1145-1150, 1976.

[13] J. G. Abbott and F. L. Thurstone, "Acoustic speckle: Theory and experimental analysis," Ultrason. Imag., vol. 1, pp. 303-324, 1979.

[14] L. J. Porcello, N. G. Massey, R. B. Innes, and J. M. Marks, "Speckle reduction in synthetic-aperture radars," J. Opt. Soc. Amer., vol. 66, no. 11, pp. 1305-1311, 1976.

[15] J. H. Hokland and T. Taxt, "Ultrasound speckle reduction using harmonic oscillator models," IEEE Trans. Ultrason., Ferroelect., Freq. Contr., vol. 41, no. 2, pp. 215-224, Mar. 1994.

[16] P. Moulin, "A wavelet regularization method for diffuse radar-target imaging and speckle-noise reduction," J. Math. Imag., Vision, vol. 3, no. 1, pp. 123-134, 1993.

[17] A. Laine, J. Fan, and W. Yang, "Wavelets for contrast enhancement of digital mammography," IEEE Eng., Med. Biol., Mag., vol. 14, no. 5, pp. 536-550, 1995

[18] S. M. Collins, D. J. Skorton, E. A. Geiser, J. A. Nichols, D. A. Conetta, N. G. Pandian, and R. E. Kerber, "Computer-assisted edge detection in two-dimensional echocardiography: Comparison with anatomic data," $J$. Amer. Soc. Echocardiogr., vol. 53, pp. 1380-1387, 1984.

[19] M. J. Lester, J. F. Brenner, and W. D. Selles, "Local transforms for biomedical image analysis," Comput. Graphics, Image Processing, vol. 13, pp. 17-30, 1980

[20] E. A. Geiser, "Edge detection and wall motion analysis," in Echocardiography: An International Review, J. Chambers and M. J. Monaghan, Eds. Oxford, U.K.: Oxford Univ. Press, 1993, pp. 71-82.

[21] X. Zong, A. F. Laine, E. A. Geiser, and D. C. Wilson, "De-noising and contrast enhancement via wavelet shrinkage and nonlinear adaptive gain," in Wavelet Applications III, Proc. SPIE, 1996, vol. 2762, pp. 566-574.

[22] S. Mallat and S. Zhong, "Characterization of signals from multiscale edges," IEEE Trans. Pattern Anal. Machine Intell., vol. 14, pp. 710-732, July 1992.

[23] X. Zong, Sub-octave wavelet representations and applications for medical image processing, Ph.D. dissertation, Univ. of Florida, Gainesville, FL., 1997.

[24] S. Zhong, Edge representation from wavelet transform maxima, Ph.D. dissertation, New York Univ., New York, 1991.

[25] D. L. Donoho and I. M. Johnstone, "Ideal spatial adaptation via wavelet shrinkage," Dept. Statistics, Stanford Univ., Stanford, CA, Tech Rep. 400, 1992.

[26] D. L. Donoho, "De-noising by soft-thresholding," IEEE Trans. Inform. Theory, vol. 41, pp. 613-627, May 1995.

[27] I. Daubechies, "Orthonormal bases of compactly supported wavelets," Commun. Pure, Appl. Math., vol. 41, no. 7, pp. 909-996, 1988.

[28] A. F. Laine and X. Zong, "A multiscale sub-octave wavelet transform for de-noising and enhancement," in Wavelet Applications in Signal and Image Processing IV, Proc. SPIE, 1996, vol. 2825, pp. 238-249.

[29] D. C. Wilson and E. A. Geiser, "Automatic center point determination in two-dimensional short-axis echocardiographic images," Pattern Recogn., vol. 25, no. 9, pp. 893-900, Sept. 1992.

[30] X. Zong, E. A. Geiser, and D. A. Conetta, "The effect of de-noising on the consistency to manually defined borders," submitted for publication. 